\title{
Dynamic multiscaling in fluid turbulence: an overview
}

\author{
Dhrubaditya Mitra ${ }^{\mathrm{a}, *}$, Rahul Pandit ${ }^{\mathrm{a}, \mathrm{b}}$ \\ ${ }^{a}$ Centre for Condensed Matter Theory, Department of Physics, Indian Institute of Science, \\ Bangalore 560012, India \\ ${ }^{\mathrm{b}}$ Jawaharlal Nehru Centre for Advanced Scientific Research, Bangalore 560 012, India
}

\begin{abstract}
We discuss the dynamic multiscaling of velocity structure functions in fluid turbulence and contrast it with the dynamic scaling of correlation functions in critical phenomena. We then outline our study of the dynamic multiscaling of velocity structure functions in the GledzerOhkitani-Yamada shell model for fluid turbulence.
\end{abstract}

Keywords: Dynamic multiscaling; Fluid turbulence

The elucidation of the scaling and universality of thermodynamic functions and correlation functions in the vicinity of a critical point was one of the highlights of statistical physics in the late 1960s and early 1970s [1]. It was shown, e.g., for an Ising ferromagnetic system near the critical point that the static spin correlation function $\Gamma$ assumes the following scaling form:

$$
\Gamma(r, t, h) \approx \frac{1}{r^{d-2+\eta}} \mathscr{F}\left(t^{v} r, h / t^{\Delta}\right) ;
$$

here $r$ is the spatial separation between the spins, $d$ the dimension of space, $t \equiv$ $\left(T-T_{c}\right) / T_{c}, h=H / k_{B} T_{c}, T$ the temperature, $T_{c}$ the critical temperature, $H$ the magnetic

\footnotetext{
is It is a great honour and pleasure to contribute this paper to the special issue of Physica A which contains the Proceedings of STATPHYS - Kolkata IV at which all participants felicitated Professor H.E. Stanley on his sixtieth birthday. He has made important contributions to several areas of statistical physics; we wish him many more years of active research in this field.
} 
field, $\xi$ the correlation length which diverges at the critical point, $\eta, v$ and $\Delta$ well-known static critical exponents, $k_{B}$ the Boltzmann constant, and $\mathscr{F}$ a scaling function which is also universal if two scale factors are suitably accounted for [1,2]. A spatial Fourier transform of Eq. (1) yields the wavevector-space version

$$
\tilde{\Gamma}(q, t, h) \approx \frac{1}{q^{2-\eta}} \mathscr{F}\left(t^{v} / q, h / t^{\Delta}\right),
$$

where $\vec{q}$ is the wavevector with magnitude $q$. Soon after this dynamic scaling was also proposed $[3,4]$ for time-dependent correlation functions in the vicinity of a critical point. In particular, the frequency-dependent version of Eq. (2) was argued to be

$$
\tilde{\Gamma}(q, \omega, t, h) \approx \frac{1}{q^{2-\eta}} \mathscr{G}\left(q^{-z} \omega, t^{\nu} / q, h / t^{\Delta}\right)
$$

here $z$ is the dynamic critical exponent, $\omega$ denotes the frequency, and $\mathscr{G}$ a scaling function. Eq. (3) also implies that, near the critical point, the relaxation time $\tau$ diverges as $\tau \sim \xi^{z}$. The systematisation of dynamic critical phenomena and the development of renormalisation groups that yield the scaling form (3) can be found in several excellent reviews and texts [1,5].

The power-law behaviours of velocity structure functions in fully developed, homogeneous, isotropic fluid turbulence, which is found at large Reynolds numbers $R e$ and far away from boundaries, have attracted several statistical physicists because of their similarity to the forms of correlation functions like $\Gamma$ near a critical point. Specifically, if we define the order- $p$, static, longitudinal velocity structure function

$$
\begin{aligned}
& S_{p}(l) \equiv\left\langle\delta v(l, t)^{p}\right\rangle, \\
& \delta v(l, t) \equiv[\vec{v}(\vec{r}+\vec{l}, t)-\vec{v}(\vec{r}, t)] \cdot(\vec{l} / l),
\end{aligned}
$$

then for $l$ in the inertial range we have

$$
S_{p}(l) \sim l^{\zeta_{p}} .
$$

Here $\vec{v}(\vec{r})$ denotes the Eulerian velocity at the spatial point $\vec{r}$ and by the inertial range we mean $L \ll l \ll \eta_{d}$, where $L$ is the length scale at which energy is pumped into the fluid and $\eta_{d}$ the spatial scale at which dissipative losses become significant; the angular brackets denote an average over the nonequilibrium statistical steady state that obtains in the turbulent fluid. The power law of Eq. (5) is reminiscent of the behaviour of correlation functions in critical phenomena (e.g., Eq. (1)); however, the simple scaling of such correlation functions must be replaced by multiscaling. For our purposes here this means that $\zeta_{p}$ is a nonlinear function of $p$ (that can also be shown to be convex); for a thorough discussion see Refs. [6,7]. The main purpose of our paper is to give a brief overview of the dynamic multiscaling of structure functions in fluid turbulence and of our recent work on this problem in the context of the Gledzer-Ohkitani-Yamada (GOY) shell model for fluid turbulence [8]. Such dynamic multiscaling has been studied much less than the dynamic scaling of correlation functions in critical phenomena; notable exceptions are the studies of Refs. [9-14].

Recall first the elegant arguments of Kolmogorov (henceforth K41) [15] that lead to the simple scaling result:

$$
\zeta_{p}^{K 41}=p / 3 .
$$


This result is based on the following assumptions (for a critical discussion see Ref. [6]): (1) The turbulence that is generated is homogeneous and isotropic and in a statistically steady state. (2) Since energy is pumped into the system at lengths $\sim L$ and dissipation is significant only for length scales $\lesssim \eta_{d}$, in the inertial range, $L \ll l \ll \eta_{d}$, all statistical quantities are independent of both the forcing term and the viscosity; energy cascades down the inertial range till it is dissipated in the dissipation range. Thus, in the inertial range $S_{p}(l)$ can depend only on $l$ and the rate of energy dissipation per unit volume per unit mass $\varepsilon$, which is assumed to be constant since experiments show that it approaches a positive, constant value at large $R e$ in three dimensions. Dimensional analysis now demands $S_{p}(l) \sim(\varepsilon l)^{p / 3}$ which leads to Eq. (6). Several experiments and direct numerical simulations (DNS) indicate that the K41 scaling result (6) does not hold $[6,7,16]$; and multiscaling deviations from it are particularly apparent for $p>3$.

The K41 analysis can be generalised easily to obtain a prediction for the dynamicscaling exponent $z$ for fluid turbulence as follows: the characteristic decay time $\tau(l)$ of a turbulent eddy at length scale $l$ can be estimated to be $\tau(l) \sim l / \delta v(l)$. Since Eq. (6) implies $\delta v(l) \sim l^{1 / 3}$ and the dynamic-scaling ansatz is $\tau(l) \sim l^{z}$, we obtain the K41 estimate for the dynamic-scaling exponent

$$
z^{K 41}=\frac{2}{3},
$$

a result that seems to go as far back as Refs. [17,18]. This value of $z^{K 41}$ emerges, either explicitly or implicitly, from field-theoretic studies of a randomly forced Navier-Stokes equation in which the variance of the stochastic force has a power-law dependence on the length scale or, equivalently, wavevector $\left(\sim k^{-3}\right.$ in three dimensions, where $k$ is the magnitude of the wavevector) [19]; these studies use Eulerian velocities and do not address the difference between the scaling of Eulerian- and Lagrangian-velocity structure functions that we discuss below.

We can also define the dynamic structure function of order- $p$ (henceforth $t$ denotes time and not the reduced temperature as in Eqs. (1)-(3))

$$
\Sigma_{p}(l, t) \equiv\left\langle[\delta v(l, 0) \delta v(l, t)]^{p / 2}\right\rangle,
$$

and extract a characteristic decay time $\tau_{p}(l)$ for $\Sigma_{p}(l, t)$ (as we describe below). The dynamic-multiscaling exponents $z_{p}$ can be defined, by analogy with dynamic critical phenomena, via the ansatz

$$
\tau_{p}(l) \sim l^{z_{p}} .
$$

Dynamic multiscaling obtains if $z_{p}$ is a nonlinear function of $p ; \mathrm{K} 41$ analysis yields $z_{p}=z^{K 41}=2 / 3$ for all $p$.

Our discussion of dynamic scaling in fluid turbulence is naive, as stated above, for reasons that have been recognised by some groups [11-14,20,21]. The crucial point is that the scaling properties of dynamic structure functions may be quite different depending on whether we use Eulerian or Lagrangian velocities because of the sweeping effect, which is implicit in early work [18]. Recall that if $\vec{X}\left(\vec{x}_{0}, t_{0} \mid t\right)$ is the position of a fluid particle at time $t$ which, at an earlier time $t_{0}$ was at position $\vec{x}_{0}$, then [22] the Lagrangian velocity $U_{\mathscr{L}}\left(\vec{x}_{0}, t_{0} \mid t\right) \equiv \partial_{t} \vec{X}\left(\vec{x}_{0}, t_{0} \mid t\right)$. It has also been suggested that the quasi-Lagrangian velocities $U_{2}\left(\vec{x}_{0}, t_{0} \mid x, t\right) \equiv \vec{v}\left(\vec{x}+\vec{X}\left(\vec{x}_{0}, t_{0} \mid t\right), t\right)$, where $\vec{v}$ denotes the Eulerian velocity, are also not affected by sweeping [11]. 
Wind-tunnel measurements [23] from 1971 showed that the shape of time-dependent, second-order correlation functions for Eulerian and Lagrangian velocities are different, even when the effect of the mean flow has been accounted for. Subsequently, it was also shown by K41-type arguments [24] that the characteristic decay time for Lagrangian and Eulerian time-dependent correlation functions should be different.

The qualitative reason for this difference is best illustrated by considering a typical experiment in which a fluid, forced through a grid or an orifice, displays homogeneous and isotropic turbulence downstream. This turbulence is advected by the mean flow. Often a probe is used to obtain a time series of the fluid velocity at a single point in space. If the mean flow velocity is large compared to the turbulent velocity fluctuations, Taylor's hypothesis [6] can be used to convert the velocity difference $\delta v(l=0, t=\mathscr{T})$ to the velocity difference $\delta v(l=\ell, t=0)$ by relating length and time via the relation $\ell=V_{m} \mathscr{T}$, where $V_{m}$ is the mean flow velocity. This linear relation between length and time scales immediately yields the dynamic-scaling exponent

$$
z_{p}^{E}=1
$$

for all $p$ if Eulerian velocities (indicated by the superscript $E$ ) are used in dynamic structure functions. It is believed that the result (10) holds even if there is no mean flow, as is typically the case in DNS of homogeneous, isotropic fluid turbulence in a cubical simulation domain with periodic boundary conditions. To see how this might arise, consider the Navier-Stokes equation

$$
\partial_{t} \vec{v}+(\vec{v} \cdot \vec{\nabla}) \vec{v}=v \nabla^{2} \vec{v}+\vec{\nabla} p / \rho+\vec{f} / \rho,
$$

where $\vec{v}, p, v$, and $\rho$ are, respectively, the Eulerian velocity, pressure, kinematic viscosity, and density of the fluid, and $\vec{f}$ is the external forcing; incompressibility, imposed by the condition $\vec{\nabla} \cdot \vec{v}=0$, is a good approximation for low-Mach-number turbulence to which we restrict ourselves. A spatial Fourier transform of Eq. (11) yields

$$
\begin{gathered}
\partial_{t} u_{l}(\vec{k}, t)+\mathrm{i} k_{m} P_{l j}(\vec{k}) \int_{\vec{p}+\vec{q}=\vec{k}} v_{j}(\vec{p}, t) v_{m}(\vec{q}, t) \mathrm{d} \vec{p} \\
=-v k^{2} u_{l}(\vec{k}, t)+\frac{\tilde{f}_{l}(\vec{k}, t)}{\rho},
\end{gathered}
$$

where $\vec{u}(\vec{k}, t)$ and $\overrightarrow{\tilde{f}}(\vec{k}, t)$ are, respectively, the spatial Fourier transform of $\vec{v}(\vec{r}, t)$ and $\vec{f}(\vec{r}, t), l, j, m$ denote Cartesian components, $i=\sqrt{-1}, \vec{k}$ is a wavevector with magnitude $k$, and incompressibility is enforced by the projection operator $P_{l j}(k)=\left[\delta_{l j}\right.$ $\left.\left(k_{l} k_{j}\right) / k^{2}\right]$. Note that the nonlinear term couples every Fourier mode directly with every other such mode. Thus large eddies, comparable to the linear size $L$ of the system, advect small eddies, with sizes corresponding to inertial-range separations. As we mentioned above, the K41 estimate for the lifetime of an inertial-range eddy of linear size $l$ is $\tau(l)=l / v(l) \sim l^{2 / 3}$. This eddy is swept past a probe in a sweeping time $\tau_{s}=l / v_{L}$, with $v_{L}$ the typical velocity of a box-size eddy. Since $v_{l} \ll v_{L}$, for $L \ll l \ll \eta_{d}$, $\tau_{s} \ll \tau(l)$ so the small eddy is swept past the probe without significant distortion. The linear relation between $\tau_{s}$ and $l$ yields Eq. (10), namely, $z_{p}^{E}=1$ for all $p$ whenever Eulerian velocities are used as is the case in most experiments and simulations. The equation of 
motion for Lagrangian velocities [22], unlike the Navier-Stokes equation (11), contains no advective nonlinear term. Thus Lagrangian velocities are unaffected by sweeping. Therefore order- $p$ dynamic structure functions of Lagrangian velocities might show multiscaling. However, this has not been checked either in numerical simulations or experiments. The DNS of Ref. [12] has shown for the Navier-Stokes equation that $z_{2}^{E}=1$ and that $z_{2}=2 / 3$, if Lagrangian velocities are used; but this work has not studied structure functions with $p>2$. Ref. [11] has advocated the use of quasi-Lagrangian velocities and suggested the following scaling relation:

$$
z_{p}=1+\zeta_{p-1}-\zeta_{p} .
$$

This has been checked to some extent [14] for the GOY shell model, not by using dynamic structure functions but by using a modified exit-time method. Studies of dynamic scaling have also been initiated for the stochastic Burgers equation [13].

Here we report briefly our numerical studies of dynamic multiscaling in the GOY shell model of fluid turbulence; a detailed discussion will be given elsewhere [25]. Our study is different from earlier work [14] in that it uses, for the first time, dynamic structure functions to obtain dynamic multiscaling exponents. As we show below, this requires very long simulations; ours are roughly a factor of $10^{4}$ longer than those of Ref. [14]. The GOY shell model [6,8,9] is defined by the following equations:

$$
\left(\frac{\mathrm{d}}{\mathrm{d} t}+v k_{n}^{2}\right) u_{n}=\mathrm{i}\left(a_{n} u_{n+1} u_{n+2}+b_{n} u_{n-1} u_{n+1}+c_{n} u_{n-1} u_{n-2}\right)^{*}+f_{n} ;
$$

here the dynamical variables are the complex, scalar velocities $u_{n}$, for the shells $n$, with one-dimensional, logarithmically spaced wavevectors $k_{n}$, i.e., $k_{n}=k_{0} 2^{n}$, and complex conjugation is denoted by $*$. The coefficients $a_{n}=k_{n}, b_{n}=-\delta k_{n-1}, c_{n}=-(1-\delta) k_{n-2}$; these are chosen to conserve the shell-model analogues of energy and helicity in the inviscid, unforced limit; and we use the standard choice $\delta=\frac{1}{2}$. By virtue of the logarithmic spacing in $k$ space one can obtain very high Reynolds number in this shell model with only a moderate number of shells as we will see below. The velocity of shell $n$ is affected directly only by the velocities of nearest- and next-nearest-neighbour shells. The forcing term $f_{n}$ is generally confined to a single shell with small $k_{n}$. Eq. (14) can be considered to be a highly simplified, one-dimensional form of the wavevector-space Navier-Stokes equation (12). Since the shell model uses scalar velocities it cannot account for vortices. Furthermore, unlike Eq. (12), the shell-model nonlinear terms allow for a direct coupling only between velocities whose shell indices differ at most by 2 . In this sense there is no sweeping effect in the shell model because velocities at the largest spatial scales cannot drive directly those at the smallest ones. It has been suggested, therefore, that one should view such shell models as very simple, quasi-Lagrangian versions of the Navier-Stokes equation [14]. This view, though appealing, cannot be substantiated by any approximate mapping of the Navier-Stokes equation onto Eq. (14). However, the form of the shell-model nonlinearity and the dissipation, which is significant at small spatial scales, suffice to lead to a cascade, as in fluid turbulence. And the static multiscaling exponents $\zeta_{p}$ for the GOY shell model defined by

$$
S_{p}(n)=\left\langle\left|u_{n}\right|^{p}\right\rangle \sim k_{n}^{-\zeta_{p}}
$$


Table 1

Order- $p$ (Column 1) static multiscaling exponents $\zeta_{p}$ (Column 2) and dynamic multiscaling exponents $z_{p}$ (Column 4) from our study of the GOY shell model (14). Column 3 contains the dynamic multiscaling exponents $z_{p}$ obtained by using Eq. (13) and the static exponents $\zeta_{p}$ in Column 2. The error estimates are obtained as described in the text.

\begin{tabular}{llll}
\hline $\operatorname{Order}(p)$ & $\zeta_{p}$ (Eq. $\left.(13)\right)$ & $z_{p}$ (our calculation) \\
\hline 1 & $0.3777 \pm 0.0001$ & $0.6221 \pm 0.0001$ & $0.60 \pm 0.02$ \\
2 & $0.7091 \pm 0.0001$ & $0.6686 \pm 0.0002$ & $0.67 \pm 0.02$ \\
3 & $1.0059 \pm 0.0001$ & $0.7030 \pm 0.0002$ & $0.701 \pm 0.009$ \\
4 & $1.2762 \pm 0.0002$ & $0.7298 \pm 0.0003$ & $0.727 \pm 0.007$ \\
5 & $1.5254 \pm 0.0005$ & $0.7511 \pm 0.0007$ & $0.759 \pm 0.009$ \\
6 & $1.757 \pm 0.001$ & $0.768 \pm 0.002$ & $0.77 \pm 0.01$ \\
\hline
\end{tabular}

are in close agreement with those found for three-dimensional fluid turbulence [9]; data for the exponents $\zeta_{p}$ from our calculations are given in Table 1.

We integrate the GOY-model Eq. (14) by using the numerical scheme of Refs. [16,26,27], with a time step $\delta t=2 \times 10^{-4}$, viscosity $v=10^{-7}$, and 22 shells, i.e., $1 \leqslant n \leqslant 22$ and $u_{n}=0$ for $n<1$ or $n>22$; and we force the first shell by setting $f_{n}=0$ for $n \geqslant 2$ and $f_{1}=(1+i) 5 \times 10^{-3}$. As in earlier studies we define the Taylor microscale $\lambda$, the integral scale $L_{\text {int }}$, the root-mean-square velocity $u_{r m s}$, the Taylor-microscale Reynolds number $R e_{\lambda}$, and the box-size eddy turnover time $\tau_{L}$ for the shell model as follows: $\lambda \equiv\left(\sum_{n}\left|u_{n}\right|^{2} / \sum_{n} k_{n}^{2}\left|u_{n}\right|^{2}\right)^{1 / 2}, L_{\text {int }} \equiv\left(\sum_{n}\left|u_{n}\right|^{2} / k_{n}^{2}\right) /\left(\sum_{n}\left|u_{n}\right|^{2} / k_{n}\right), u_{r m s} \equiv$ $\left(2 \sum_{n}\left|u_{n}\right|^{2}\right)^{1 / 2}, R e_{\lambda} \equiv \lambda u_{r m s} / v$, and $\tau_{L} \equiv L_{\text {int }} / u_{r m s}$. For the parameters we use here we find $R e_{\lambda} \simeq 2 \times 10^{5}$ and $\tau_{L} \simeq 10^{6} \delta t$. Data from the first $T_{t r}$ time steps are discarded so that transients can die down and the system can achieve a nonequilibrium statistical steady state; we use $T_{t r}=5 \times 10^{4} \tau_{L}$. We then average our data for dynamic structure functions for $T_{a v}=10^{5} \tau_{L}$, which is larger by a factor of roughly $10^{4}$ than the simulations of Ref. [14].

By analogy with Eq. (8) we define the order- $p$ complex dynamic structure function for the GOY model (14) as

$$
C_{n}^{p}(t) \equiv\left\langle\left[u_{n}(t) u_{n}^{*}(0)\right]^{p / 2}\right\rangle \text {; }
$$

this yields the order- $p$ static structure function (15) at $t=0$. It is convenient to work with normalised structure function

$$
\mathscr{C}_{n}^{p}(t)=\frac{C_{n}^{p}(t)}{C_{n}^{p}(0)}
$$

Our results show that the imaginary part of $\mathscr{C}_{n}^{p}(t)$ is negligibly small compared to its real part. Therefore we work with the real part of $\mathscr{C}_{n}^{p}(t)$, namely, $F_{n}^{p}(t) \equiv \mathfrak{R}\left[\mathscr{C}_{n}^{p}(t)\right]$. Our results remain unchanged if we use the absolute value of $\mathscr{C}_{n}^{p}(t)$ instead. We use the order- $p$ generalisation of the integral time scale of [23] and define the order- $p$ integral time scale as

$$
T_{n}^{p}(\mu)=\int_{0}^{T_{\mu}} F_{n}^{p}(t) \mathrm{d} t,
$$


where $T_{\mu}$ is chosen such that $F_{n}^{p}\left(T_{\mu}\right)=\mu$, for all $n$, and $0<\mu<1$. We have checked that our results for the dynamic exponents $z_{p}$ do not depend significantly on $\mu$ for $0.3 \leqslant \mu \leqslant 0.7$. The wavevector-space version of the dynamic-multiscaling ansatz is

$$
T_{n}^{p} \sim k_{n}^{-z_{p}}
$$

for $\left(k_{n}\right)^{-1}$ in the inertial range. Hence, the slope of a $\log -\log$ plot of $T_{n}^{p}$ versus $k_{n}$ yields the dynamic multiscaling exponent $z_{p}$. We carry out such slope calculations with 50 different initial conditions and obtain 50 estimates for $z_{p}$. The values given in Table 1 are the means of these 50 estimates; and the errors indicate the standard deviations. The values that we obtain for $z_{p}$ (Column 3 Table 1) are consistent with those that follow from the relation (13) (Column 2 Table 1) if we use the static multiscaling exponents $\zeta_{p}$ we have calculated (Column 1 Table 1). In Table 1 the errors in Column 1 are used to calculate those in Column 2.

In summary, then, we have obtained the dynamic multiscaling exponents for the GOY shell model for fluid turbulence up to order $p=6$ by using dynamic structure functions. The clear evidence we find for dynamic multiscaling confirms the absence of direct sweeping effects in the GOY shell model. Our results agree well with the relation (13). A detailed comparison of our work with the exit-time analysis of Ref. [14] will be given elsewhere [25]. We are also carrying out a similar analysis of dynamic structure functions for the Navier-Stokes equation, with Eulerian, Lagrangian, and quasi-Lagrangian velocities. We hope that our work will stimulate experimental studies of such structure functions, now that high-Reynolds-number Lagrangian measurements of fluid turbulence are increasing [28].

We thank A. Celani, S.K. Dhar, U. Frisch, C. Jayaprakash, and A. Sain for discussions. This work was supported by the Indo-French Centre for the Promotion of Advanced Research (IFCPAR Project No. 2402-2). D.M. thanks the Council of Scientific and Industrial Research, India for support.

\section{References}

[1] P.M. Chaikin, T.C. Lubensky, Principles of Condensed Matter Physics, Cambridge University Press, Cambridge, 1998.

[2] D. Stauffer, M. Ferer, M. Wortis, Phys. Rev. B 6 (1972) 3426;

A. Aharony, Phys. Rev. B 9 (1974) 2107.

[3] R.A. Ferrel, N. Menyhárd, H. Schmidt, F. Schwabl, P. Szépfalusy, Phys. Rev. Lett. 18 (1967) 891; R.A. Ferrel, N. Menyhárd, H. Schmidt, F. Schwabl, P. Szépfalusy, Ann. Phys. (N.Y.) 47 (1968) 565.

[4] B.I. Halperin, P.C. Hohenberg, Phys. Rev. Lett. 19 (1967) 700; B.I. Halperin, P.C. Hohenberg, Phys. Rev. 177 (1969) 952.

[5] P.C. Hohenberg, B.I. Halperin, Rev. Mod. Phys. 49 (1977) 435.

[6] U. Frisch, Turbulence: the Legacy of A.N. Kolmogorov, Cambridge University Press, Cambridge, 1996.

[7] K.R. Sreenivasan, R.A. Antonia, Ann. Rev. Fluid Mech. 29 (1997) 435.

[8] E.B. Gledzer, Sov. Phys. Dokl. 18 (1973) 216; K. Ohkitani, M. Yamada, Prog. Theor. Phys. 81 (1989) 329.

[9] L.P. Kadanoff, D. Lohse, J. Wang, R. Benzi, Phys. Fluids 7 (1995) 617.

[10] O. Gat, I. Procaccia, R. Zeitak, Phys. Rev. E 51 (1995) 1148.

[11] V.S. L’vov, E. Podivilov, I. Procaccia, Phys. Rev. E 52 (1997) 7030. 
[12] Y. Kaneda, T. Ishihara, K. Gotoh, Phys. Fluids 11 (1999) 2154.

[13] F. Hayot, C. Jayaprakash, Phys. Rev. E 57 (1998) R4867.

[14] L. Biferale, G. Bofetta, A. Celani, F. Toschi, Physica D 127 (1999) 187.

[15] A.N. Kolmogorov, Dokl. Akad. Nauk USSR 30 (1941) 9.

[16] S.K. Dhar, A. Sain, R. Pandit, Phys. Rev. Lett. 78 (1997) 2964.

[17] L. Onsager, Phys. Rev. 68 (1945) 286 (abstract only);

L. Onsager, Nuovo Cimento 9 (suppl.) (1949) 279.

[18] W. Heisenberg, Z. Phys. 124 (1948) 628;

C.F. von Weizsäcker, Z. Phys. 124 (1948) 614.

[19] C. DeDominicis, P.C. Martin, Phys. Rev. A 19 (1979) 419;

V. Yakhot, S.A. Orszag, Phys. Rev. Lett. 57 (1986) 1722;

J.K. Bhattacharjee, Phys. Fluids A 3 (1991) 768;

J.K. Bhattacharjee, J. Phys A 27 (1994) L347.

[20] Y. Kaneda, Phys. Fluids A 5 (1993) 2835.

[21] T. Gotoh, R.S. Rogallo, J.R. Herring, R.H. Kraichnan, Phys. Fluids A 5 (1993) 2846.

[22] S.B. Pope, Turbulent Flows, Cambridge University Press, Cambridge, 2000.

[23] G. Comte-Bellot, S. Corrsin, J. Fluid Mech. 48 (1971) 273;

D. Shlien, S. Corrsin, J. Fluid Mech. 62 (1974) 255.

[24] H. Tennekes, J. Fluid Mech. 67 (1975) 561.

[25] D. Mitra, R. Pandit, to be published.

[26] D. Pisarenko, L. Biferale, D. Courvoisier, U. Frisch, M. Vergassola, Phys. Fluids A 5 (1993) 2533.

[27] S.K. Dhar, Ph.D. Thesis, Indian Institute of Science, Bangalore, 1996, unpublished.

[28] S. Ott, J. Mann, J. Fluid Mech. 422 (2000) 207;

A. La Porta, G.A. Voth, A.M. Crawford, J. Alexander, E. Bodenschatz, Nature (London) 409 (2001) 1017 ;

N. Mordant, P. Metz, O. Michel, J.-F. Pinton, Phys. Rev. Lett. 87 (2001) 214501. 